\title{
AR-42: A Pan-HDAC Inhibitor with Antitumor and Antiangiogenic Activities in Esophageal Squamous Cell Carcinoma
}

This article was published in the following Dove Press journal: Drug Design, Development and Therapy

\author{
Yuxuan Zhu* \\ Ting Yuan* \\ Yuan Zhang \\ Jianyou Shi \\ Lan Bai \\ Xingmei Duan \\ Rongsheng Tong \\ Lei Zhong
}

Personalized Drug Therapy Key Laboratory of Sichuan Province, Department of Pharmacy, Sichuan Provincial People's Hospital, University of Electronic Science and Technology of China, Chengdu, Sichuan 610072,

People's Republic of China

*These authors contributed equally to this work
Purpose: Esophageal squamous cell carcinoma (ESCC) is a refractory malignancy with high morbidity and mortality. Thus, there is an urgent need to find effective targets and agents for ESCC treatment. The purpose of this study was to assess the anti-ESCC effects of a pan-histone deacetylase (HDAC) inhibitor AR-42 and its mechanisms of action.

Methods: Immunohistochemical staining was performed to detect HDAC1 expression in ESCC and adjacent tissue samples. MTT assay, Edu cell proliferation test, flow cytometry, and subcutaneous xenograft were used to assess the anti-ESCC effects of AR-42; furthermore, the antiangiogenic activity of AR-42 was evaluated using endothelial cell migration, invasion, and tube formation assays as well as zebrafish angiogenesis assay. Western blot analysis was performed to explore the underlying mechanism of the anti-ESCC activity of AR-42.

Results: HDAC1-positive expression was much higher in ESCC cells than in paracancerous tissues, and the elevated HDAC1 expression was a strong indicator of lymph node metastasis and a more advanced TNM stage of ESCC. Moreover, AR-42 potently suppressed ESCC cell growth through cellular proliferation inhibition and apoptosis induction. Moreover, AR-42 displayed a moderate antiangiogenic activity, and it could significantly inhibit the migration, invasion and tubulogenesis of human umbilical vein endothelial cells as well as intersegmental vessel formation in zebrafish at micromolar concentrations. More importantly, the inhibitory activity of AR-42 on ESCC cells and angiogenesis could also be observed in the TE-1 xenograft model. Further studies showed that AR-42 exerts its anti-ESCC effects mainly by upregulating the expression of p21 and blocking the transduction of multiple signaling cascades related to tumor growth, especially Stat3-mediated signaling.

Conclusion: Overall, AR-42 has significant potency for inhibiting ESCC cell growth and shows moderate effect in suppressing angiogenesis, displaying strong anti-ESCC effects in vitro and in vivo. Thus, AR-42 deserves further evaluation as a potential candidate for ESCC therapy.

Keywords: esophageal squamous cell cancer, AR-42, histone deacetylase, angiogenesis

\section{Introduction}

Esophageal cancer is a common malignant tumor and ranks seventh in incidence and sixth in mortality worldwide. ${ }^{1}$ Squamous cell carcinoma and adenocarcinoma are the two predominant histologic subtypes of esophageal cancer, with the former accounting for $>90 \%$ of all cases of esophageal cancer. ${ }^{1,2}$ Although the 5-year global survival rate of esophageal squamous cell carcinoma (ESCC) patients (30-40\%) has improved due to the advancements in diagnostic and treatment modalities, the cancer is still detected in advanced stages in many patients and the efficacy of current systematic treatments, especially
Correspondence: Lei Zhong; Rongsheng Tong

of Sichuan Province, Department of Pharmacy, Sichuan Provincial People's Hospital, University of Electronic Science and Technology of China, No. 32

West Second Section First Ring Road Chengdu, Sichuan 610072, People's Republic of China

Tel +86 I3458580284;

$+8628-87393405$

Email zhl7235301@I63.com;

1505855365@qq.com 
chemotherapy, remains unsatisfactory. ${ }^{2,3}$ Therefore, developing novel therapeutic strategies and agents remains an urgent need for ESCC therapy.

Accumulating evidence has suggested that epigenetic regulation is involved in tumor occurrence and progression through affecting DNA methylation and histone post-translational modification, thereby influencing the chromatin structure and oncogene or tumor suppressor gene expression. ${ }^{4,5}$ Histone deacetylases (HDACs) determine the status of histone acetylation, which is an important epigenetic modification related to the development of numerous malignancies, including hepatocellular carcinoma, pancreatic cancer, lung cancer, and hematological malignancies. ${ }^{6-9}$ Inhibition of HDACs has become a potential treatment strategy for these cancers. Zeng et al reported that in 86 paired ESCC samples, HDAC4 expression remarkably increased in tumors than in the paired normal tissues. Furthermore, HDAC4 inhibition led to decreased vimentin and increased E-cadherin expression, which are important features of EMT suppression. ${ }^{10} \mathrm{Li}$ et al indicated that HDAC2 expression was higher in ESCC tissues than in the normal esophageal mucosa and atypical hyperplasia tissues and was closely related to unfavorable clinicopathologic characteristics including a deep invasion depth and lymph node metastasis. HDAC2 downregulation with small interfering RNA may effectively inhibit ESCC cell growth. ${ }^{11}$ Moreover, it has been reported that HDAC1 inhibition by shRNA may efficaciously restrain ESCC cell growth, migration, and invasion and increase the sensitivity of tumor cells to cytotoxic chemotherapeutic agents. ${ }^{12}$ Thus, it can be seen that HDACs are also potential therapeutic targets for ESCC.

The present study aimed to detect HDAC1 expression in and around ESCC tissues and comprehensively assess the anti-ESCC effects of AR-42, a phenylbutyrate-derived pan-HDAC inhibitor with low nanomolar IC50s against HDACs including HDAC $1 .{ }^{13-17}$

\section{Materials and Methods}

\section{Tissue Samples}

ESCC and adjacent tissue samples were obtained from the Sichuan Provincial People's Hospital. This study was approved by the hospital's Ethics Review Committee and informed consent was also signed by relevant patients. All paraffinembedded samples were sliced for HDAC1 (Abcam, Cambridge, MA, USA) immunohistochemical staining. HDAC1 expression was then scored by Yuan Zhang and Rongsheng Tong according to the staining depth: no dyeing, "1"; pale yellow dyeing, "2"; yellow dyeing, "3"; and deep yellow or brown dyeing, " 4 ". Both scores 3 to 4 denote that the sample is positive for HDAC1 expression.

\section{Cell Lines and Reagents}

Eca109 and TE-1 ESCC cells were obtained from the National Platform of Experimental Cell Resources for Sci-Tech (Shanghai, China), and maintained in RPMI-1640 complete medium (Gibco, Eggenstein, Germany). Human umbilical vein endothelial cells (HUVECs) were isolated from the umbilical cord with written informed consent by a parturient, and the experimental protocol was approved by the ethics committee of Sichuan Provincial People's Hospital. HUVECs were grown in EBM-2 medium supplemented with EndoGRO ${ }^{\mathrm{TM}}$ VEGF supplement kit (Millipore, Billerica, MA, USA). All cell lines were cultured at $37^{\circ} \mathrm{C}$ in a $\mathrm{CO}_{2}$ incubator. AR-42 and other positive agents were all purchased from Target Molecule Corporation (Boston, MA, USA).

\section{MTT Assay}

MTT assay was performed to detect cell viability. Briefly, ESCC cell lines seeded in 96 well plates were incubated with AR-42 across a 9-dose range from $0.001 \mu \mathrm{M}$ to $10 \mu \mathrm{M}$. After $72 \mathrm{~h}$ of treatment, cell viability was detected by incubating the plates for $3 \mathrm{~h}$ with $5 \mathrm{mg} / \mathrm{mL}$ MTT solution (MilliporeSigma, Burlington, MA, USA) at $37^{\circ} \mathrm{C}$, followed by dissolving the formazan crystals with $20 \%$ acidified SDS (Solarbio, Beijing, China) overnight. Optical density at $570 \mathrm{~nm}$ was determined using MK3 multiskan spectrum microplate spectrophotometer (Thermo Scientific, Waltham, MA, USA).

\section{Colony Formation Test}

ESCC cell lines seeded in 12 well plates were incubated with AR-42 or a vehicle for 12 days. The medium containing AR42 was replaced every 3 days. Then the supernatant was removed, and $0.05 \%$ crystal violet (Beyotime, Shanghai, China) was added to stain the adherent viable cells.

\section{Cell Proliferation Test}

ESCC cells cultured in 96-well plates were treated with AR42 for $18 \mathrm{~h}$. EdU-Apollo incorporation assay was performed following RIBOBIO's kit manual (Guangzhou, Guangdong, China) to determine the cell proliferation activity.

\section{Apoptosis Assay}

ESCC cells cultured in 6-well plates were treated with AR-42 for $24 \mathrm{~h}$, and then harvested for apoptosis test in flow cytometer following KeyGEN's Annexin V/PI double staining kit manual (Nanjing, Jiangsu, China). 


\section{Scratch Wound Assay}

HUVECs were cultured in 24-well plates at $37^{\circ} \mathrm{C}$ until confluence. Then the cells were scratched with the tip of a yellow pipette and incubated with AR- 42 for $18 \mathrm{~h}$. Pictures were taken immediately after wounding and after $18 \mathrm{~h}$ of incubation using an OLYMPUS inverted microscope (Shinjuku-ku, Tokyo, Japan). The migrating cells were then quantified using Image J software (National Institutes of Health, Bethesda, MD, USA).

\section{Transwell Chamber Assay}

The transwell compartments pre-inserted in 24-well plates were coated with diluted Matrigel (BD Biosciences, San Jose, CA, USA). Endothelial cells were seeded in the upper chambers in the AR-42-containing EBM-2 medium, and the lower chambers were filled with EGM-2 complete medium. Invasion was terminated after treatment with AR-42 for 24 $h$ by removing the unmigrated cells in the upper chambers of transwell compartments with cotton swabs. The invading cells in the lower chambers were labeled with crystal violet and captured under a Leica microscope (Solms, Germany) as well as quantified using Image $\mathbf{J}$ software (National Institutes of Health, Bethesda, MD, USA).

\section{Tube Formation Assay}

HUVECs were cultured in 96-well plates pre-coated with Matrigel (BD Biosciences, San Jose, CA, USA), and then incubated with the indicated concentrations of AR- 42 or vehicle the next day. After treatment with agents at $37^{\circ} \mathrm{C}$ for $6 \mathrm{~h}$, the formation of a network of vessel-like tubes was captured under an OLYMPUS inverted microscope (Shinjuku-ku, Tokyo, Japan), and the vessel branch points were quantified by manual counting.

\section{Zebrafish Angiogenesis Assay}

FLK-1 promoter EGFP transgenic zebrafish was used to further evaluate the antiangiogenic effect of AR-42, as reported previously. ${ }^{18}$ Briefly, 15 -h post fertilization, zebrafish embryos were incubated in Holtfreter's solution in the presence of vehicle, AR-42 or positive-control sorafenib. Then $0.01 \%$ tricaine (Aladdin, Shanghai, China) was used to anesthetize the zebrafish, and their intersegmental blood vessels were captured using a fluorescence microscope (Carl Zeiss Meditec AG, Jena, Germany).

\section{Western Blotting}

AR-42 and DMSO vehicle-treated ESCC cells were harvested after incubation for $18 \mathrm{~h}$ and lysed in RIPA extraction reagent
(Solarbio, Beijing, China). SDS-PAGE was performed to separate equivalent amounts of total protein from the cell extracts. Proteins of interest were transferred to polyvinylidene difluoride membranes (Millipore, Billerica, MA, USA) and then examined by immunoblotting with the corresponding primary antibodies and horseradish peroxidase-coupled secondary antibodies following the antibody specifications. All antibodies were obtained from CST (Boston, MA, USA) or Abcam (Cambridge, MA, USA). The protein blots were developed by ECL detection system of Millipore.

\section{Subcutaneous Xenograft Study}

Animal studies were performed in accordance with the 2017 guideline on administration of laboratory animal in China and other related regulations in Animal Welfare Act. The experimental scheme was approved by the Animal Care and Use Committee of Sichuan Provincial People's Hospital. TE-1 cells $\left(5 \times 10^{6} / 100 \mu \mathrm{L}\right)$ were suspended in a serum-free medium, and subcutaneously implanted into NOD/SCID mice (Charles River, Beijing, China). Once the mean tumor volume reached $150 \mathrm{~mm}^{3}$, the mice were randomized into vehicle, $25 \mathrm{mg} / \mathrm{kg}$, and $50 \mathrm{mg} / \mathrm{kg}$ groups and administered by oral gavage every other day. Tumor sizes and mice weight were measured using a vernier caliper and an electronic balance, respectively. Tumor volume was calculated as $0.5 \times$ width $^{2} \times$ length.

\section{Immunohistochemistry}

For immunohistochemistry studies, the implanted tumors were resected from mice after a 21-day drug treatment and fixed with formalin. The tumor tissues were then embedded in paraffin, and tumor sections were immunostained with histone H3 (acetyl K9), Ki67, TUNEL, or CD31 (Abcam, Cambridge, MA, USA) according to standard protocols.

\section{Statistical Analysis}

Data were analyzed using Pearson's chi-square test using R v3.3.1 software and Student's $t$-test using GraphPad v5.0 software (GraphPad Software, Inc., La Jolla, CA, USA). Differences were considered significant for $P$ values $<0.05$.

\section{Results HDACI Expression Status in ESCC and Adjacent Tissues}

A total of 62 ESCC tissues and 55 adjacent non-tumor tissues (ANTTs) were collected for HDAC1 immunostaining. The representative images of HDACl expression in both tissues are shown in Figure 1A. HDAC1-positive expression was 

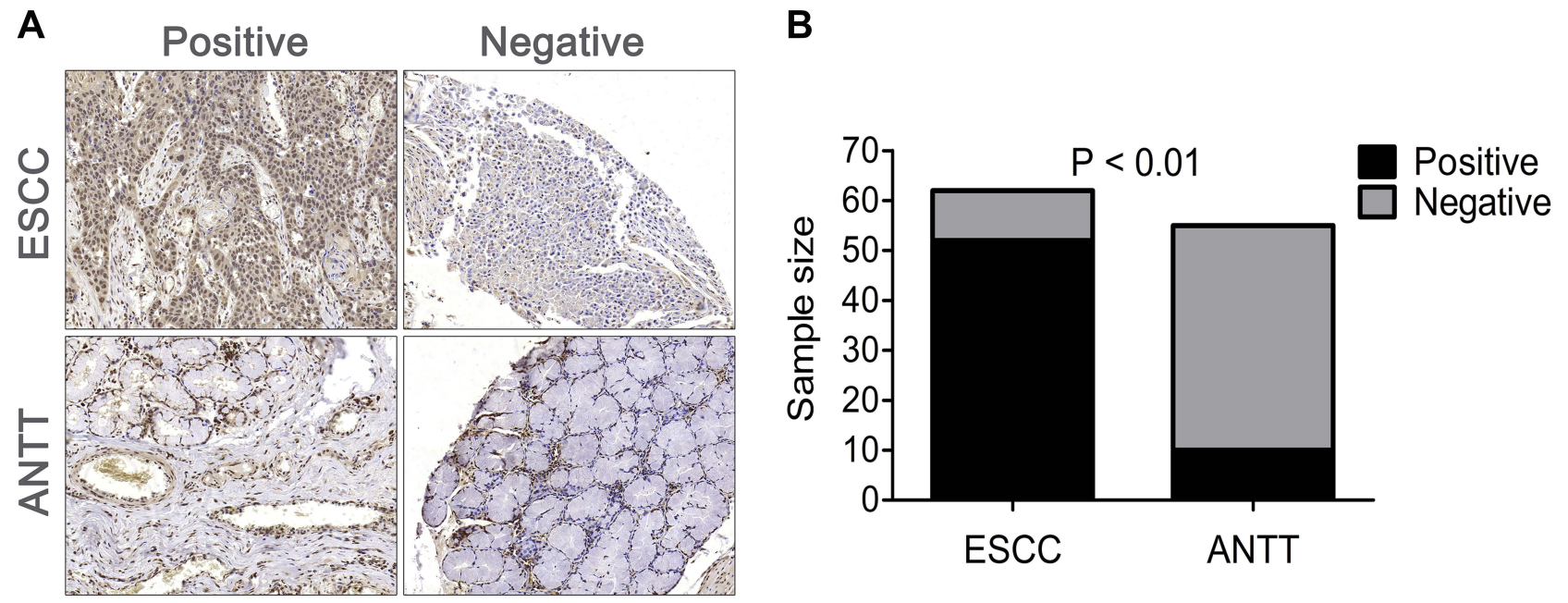

Figure I HDACI expression in human ESCC and ANTT. (A) HDACI immunohistochemical staining in ESCC cells and adjacent non-tumor tissues (ANTTs) (20x). (B) Statistical analysis of $\mathrm{HDACl}$-positive expression in ESCC and ANTT.

Abbreviations: $\mathrm{HDACl}$, histone deacetylase I; ESCC, esophageal squamous cell carcinoma; ANTT, adjacent non-tumor tissue.

prominently higher in ESCC tissues (83.9\%) than in ANTTs (18.2\%) (Figure 1B). We further assessed the relevance of HDAC1-positive expression for clinicopathologic features of ESCC and found a significant association between HDAC1positive expression and lymph node metastasis as well as a more advanced TNM stage $(P<0.01$; Table 1$)$. These results suggest that HDAC1 is closely related to ESCC progression and may be a promising target for ESCC treatment.

\section{AR-42 Restrained ESCC Cell Growth in vitro} AR-42 (Figure 2A) is a pan-HDAC inhibitor with low nanomolar $\mathrm{IC}_{50} \mathrm{~s}$ against HDACs including HDAC1. As depicted

Table I The Association of Positive HDACI Expression with Clinicopathological Characteristics of ESCC Patients

\begin{tabular}{|l|l|l|l|l|l|}
\hline $\begin{array}{l}\text { Clinicopatho- } \\
\text { logical Features }\end{array}$ & Total & \multicolumn{2}{|l|}{ HDACI } & $\begin{array}{l}\text { Positive } \\
\text { Rate } \\
(\%)\end{array}$ & \\
\cline { 3 - 6 } & & Positive & Negative \\
\hline $\begin{array}{l}\text { Gender } \\
\text { Male }\end{array}$ & 47 & 40 & 7 & 85.1 & 0.6929 \\
Female & 15 & 12 & 3 & 80.0 & \\
\hline $\begin{array}{l}\text { Age } \\
\text { S60 }\end{array}$ & 34 & 28 & 6 & 82.4 & 1 \\
$>60$ & 28 & 24 & 4 & 85.7 & \\
\hline $\begin{array}{l}\text { TNM staging } \\
\text { I+II }\end{array}$ & 13 & 4 & 9 & 30.8 & $<0.01$ \\
III+IV & 49 & 48 & 1 & 98.0 & \\
\hline $\begin{array}{l}\text { Lymph node } \\
\text { metastasis }\end{array}$ & & & & & \\
Yes & 42 & 39 & 3 & 92.8 & $<0.01$ \\
No & 20 & 13 & 7 & 65.0 & \\
\hline
\end{tabular}

in Figure 2B, AR-42 effectively restrained the viability of ESCC cells Eca109 and TE-1 in MTT assay with $\mathrm{IC}_{50}$ values of $0.44 \mu \mathrm{M}$ and $0.28 \mu \mathrm{M}$, respectively, which is slightly more potent than that of another approved pan-HDAC inhibitor vorinostat (Corresponding IC50 values for vorinostat are $0.91 \mu \mathrm{M}$ and $0.78 \mu \mathrm{M}$, respectively). As a commonly used clinical chemotherapy drug for ESCC, cisplatin inhibited ESCC cell viability at micromolar concentrations. In addition, the clone formation test was performed to evaluate the antiESCC activity after long-term treatment with AR-42. The colonies of both ESCC cells decreased dose-dependently after being treated with AR-42 (Figure 2C).

To further clarify the specific mode of action of AR-42 against ESCC cell growth, EdU incorporation assay and Annexin V/PI double staining were performed to detect ESCC cellular proliferation and apoptosis, respectively. As shown in Figure 3A, AR-42 significantly reduced the number of red-stained nuclei (proliferating cells) at $1 \mu \mathrm{M}$ concentration in both Eca109 and TE-1 cells, exhibiting strong antiproliferative ability against ESCC cells. Meanwhile, AR-42 dose-dependently increased the total percentage of Eca109 in apoptotic cells (defined as the sum of both late and early apoptotic cells), with apoptotic rates of $16 \% \pm 1.2 \%, 37 \% \pm 1.7 \%$, and $41 \% \pm 1.7 \%$ for $0.3 \mu \mathrm{M}, 1 \mu \mathrm{M}$, and $3 \mu \mathrm{M}$ concentration treatment groups, respectively (Figure 3B). The pro-apoptotic activity of AR-42 was also detected in TE-1 cells, in which the Annexin V-positive populations markedly increased after exposure to AR-42 for $24 \mathrm{~h}$ even at 

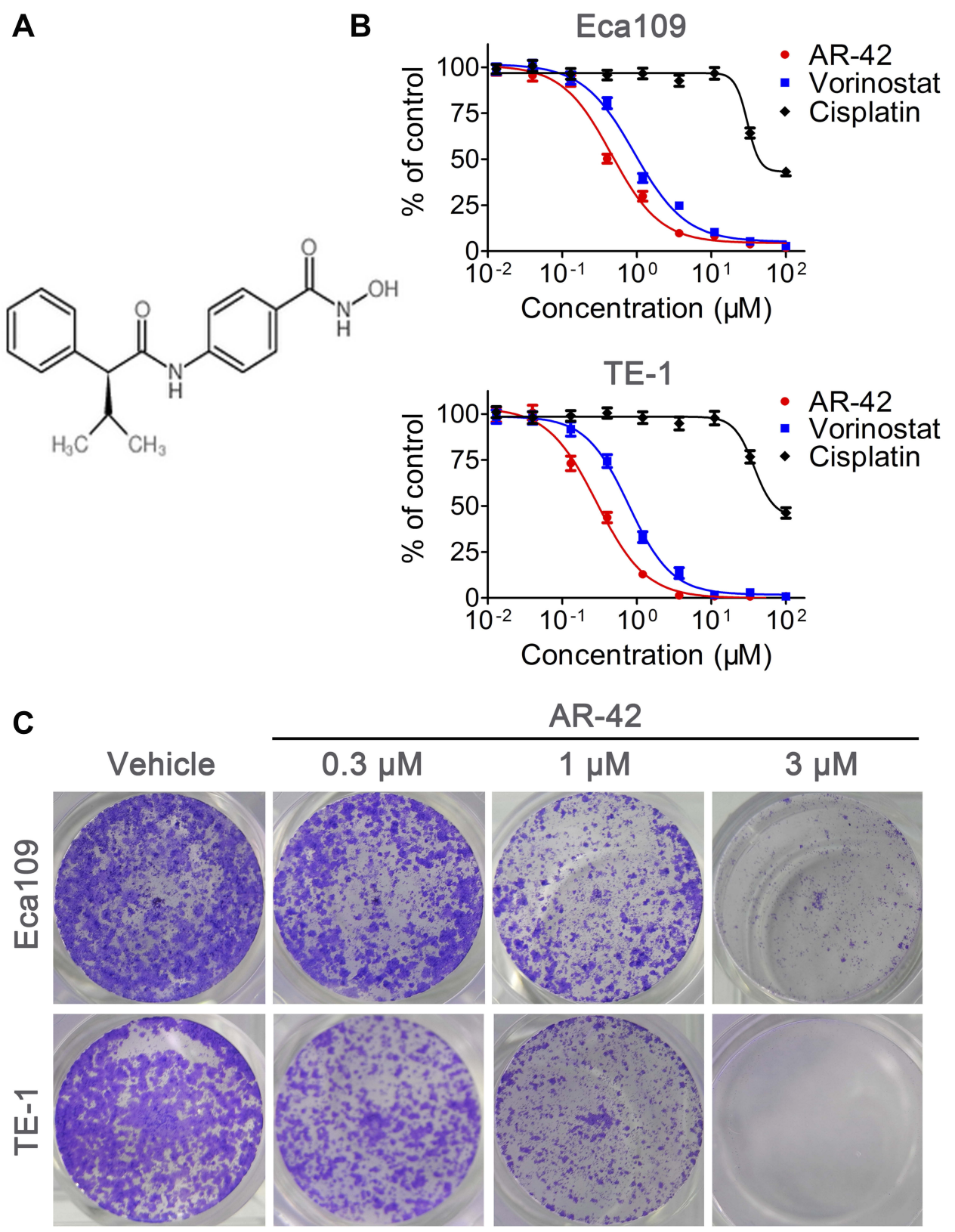

Figure 2 AR-42 inhibits ESCC cell viability in vitro. (A) Chemical structure of AR-42. (B) The sensitivity of ESCC cells to AR-42 was detected using MTT test. Cell viability is presented as mean $\pm S D(n=3)$. (C) AR-42 inhibited the formation of Ecal 09 and TE-I cell clones.

Abbreviation: ESCC, esophageal squamous cell carcinoma.

a relative low concentration (Figure 3B). These results suggest that AR-42 possesses strong anti-ESCC activity in vitro, and its potency is mainly attributable to proliferation inhibition and apoptosis induction.

\section{Anti-Angiogenesis Effect of AR-42}

Tumor angiogenesis involves the migration and invasion of endothelial cells and is a complex process. As shown in Figure 4A, AR-42 reduced the number of migrating cells dose-dependently in the scratch assay, with an $\mathrm{IC}_{50}$ of about $2 \mu \mathrm{M}$. In endothelial cell invasion assay, the invading cells evidently decreased in the AR-42-treated groups compared with those in the control (Figure 4B). Moreover, tube formation of HUVECs on Matrigel was also evaluated by counting the branching points of the tubular network. As depicted in Figure 4C, treatment with AR-42 could also efficaciously suppress endothelial tubulogenesis at concentrations $\geq 1.25 \mu \mathrm{M}$.

The anti-angiogenesis ability of AR-42 was further evaluated using the transgenic zebrafish model, with the 
A

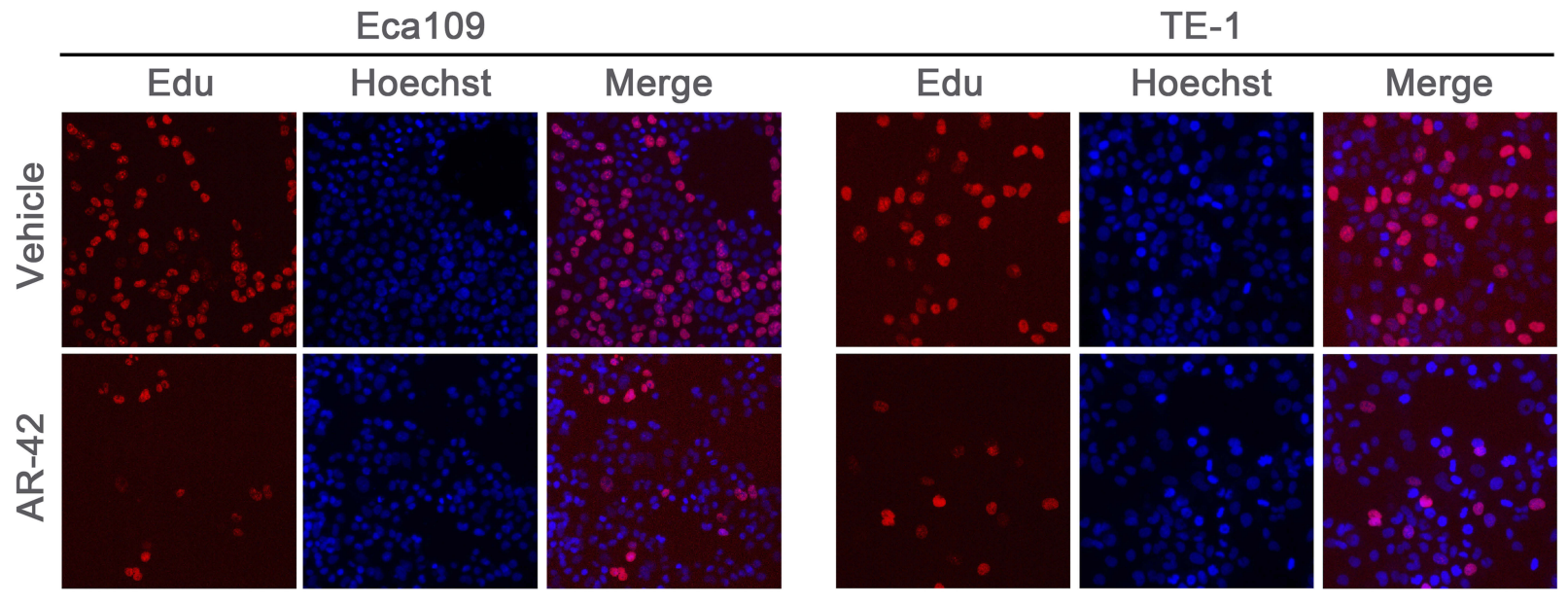

B
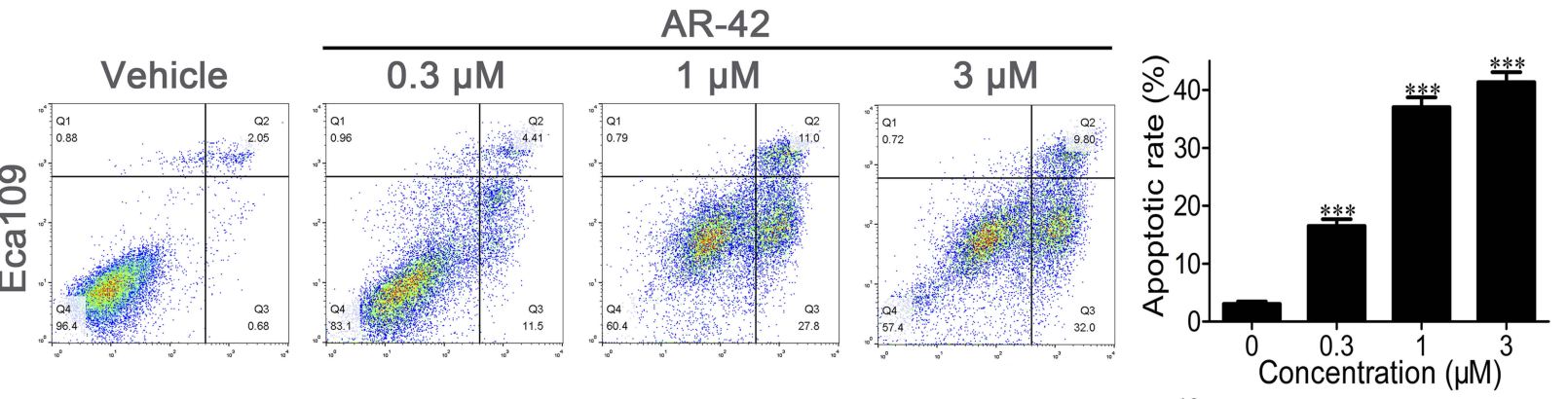

$\overline{\mathbf{0}}$
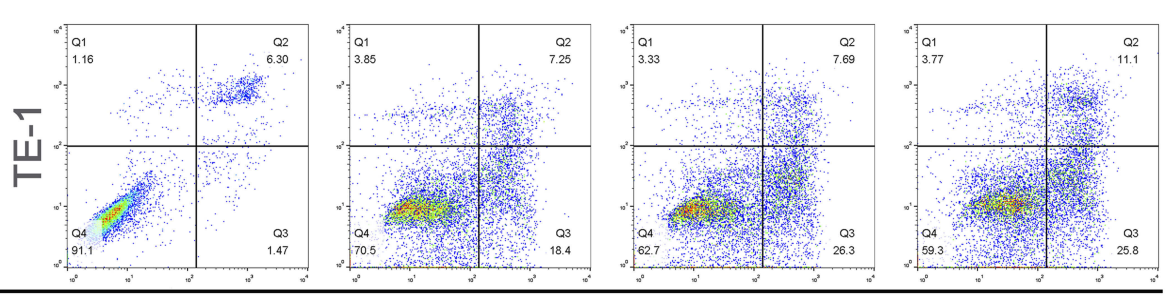

Annexin V-FITC

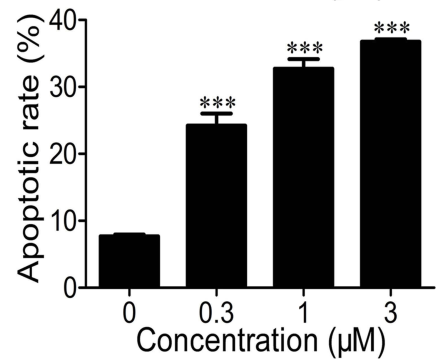

Figure 3 Anti-proliferation and pro-apoptosis effects of AR-42 in vitro. (A) Cellular proliferation test was performed in ESCC cells after treatment with AR-42 (I $\mu$ M) for I8h. (B) Annexin $\mathrm{V} / \mathrm{PI}$ double staining was performed in ESCC cells after treatment with the indicated concentrations of AR-42 for $24 \mathrm{~h}$. Apoptotic rate is quantified for statistics. $* * * \mathrm{P} P<0.00 \mathrm{I}$ vs vehicle. Abbreviation: ESCC, esophageal squamous cell carcinoma.

antiangiogenic agent sorafenib used as a positive control. AR42 at $1.25 \mu \mathrm{M}$ had little antiangiogenic potency in zebrafish, but dose-dependent inhibition could be observed at concentrations $>2.5 \mu \mathrm{M}$. Furthermore, the anti-angiogenesis activity of AR-42 was weaker than that of sorafenib; the latter could almost completely suppress the intersegmental angiogenesis in zebrafish at $2.5 \mu \mathrm{M}$ (Figure 4D). Overall, these results prove that AR-42 possesses moderate anti-angiogenesis ability in vitro and in vivo.

\section{Anti-ESCC Mechanisms of Action of AR-42}

The anti-ESCC molecular mechanisms of AR-42 were explored using an immunoblot assay. The results showed that AR-42 strikingly elevated the acetylation level of histone
H3 (Lys 9) (HDAC1 substrate) with little effect on HDAC1 expression (Figure 5), suggesting that it acts primarily by inhibiting HDAC1 activity rather than HDAC1 expression. The cell cycle inhibitor p21 is frequently epigenetically silenced by HDACs in tumors, whereas the low expression of p21 was reversed after treatment with pan-HDAC inhibitor AR-42 in both Eca109 and TE-1 cells (Figure 5); this is one of the contributors for the anti-ESCC effects of AR-42. Moreover, the activity of several pivotal signaling pathways associated with tumor growth was also detected in AR-42 treated ESCC cells. As shown in Figure 5, Stat3 phosphorylation (Tyr705) was effectively suppressed by AR-42 at concentrations $\geq 0.3 \mu \mathrm{M}$ in both ESCC cell lines, whereas p-Akt (Ser473) and p-ERK (Thr202/Tyr204) could just be inhibited 
A
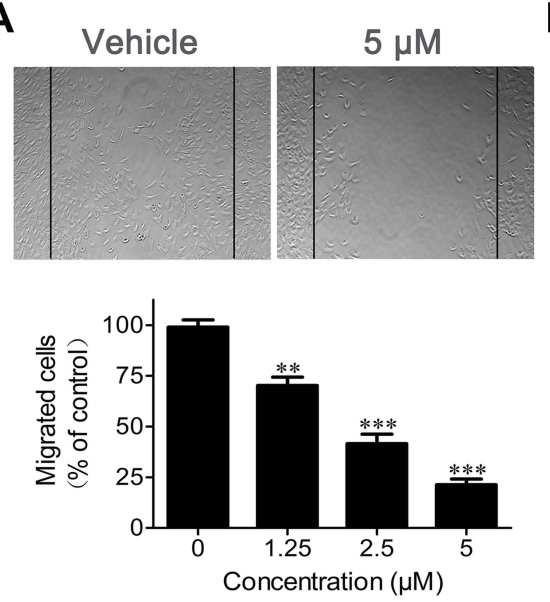

B
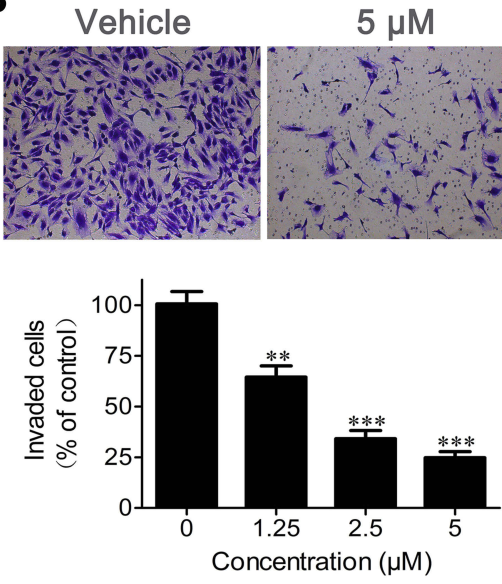

C
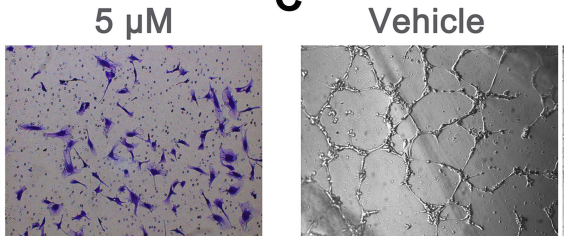

$5 \mu \mathrm{M}$

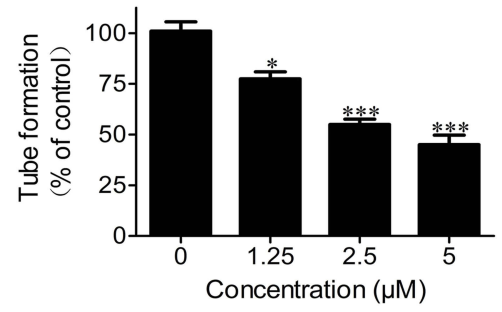

D
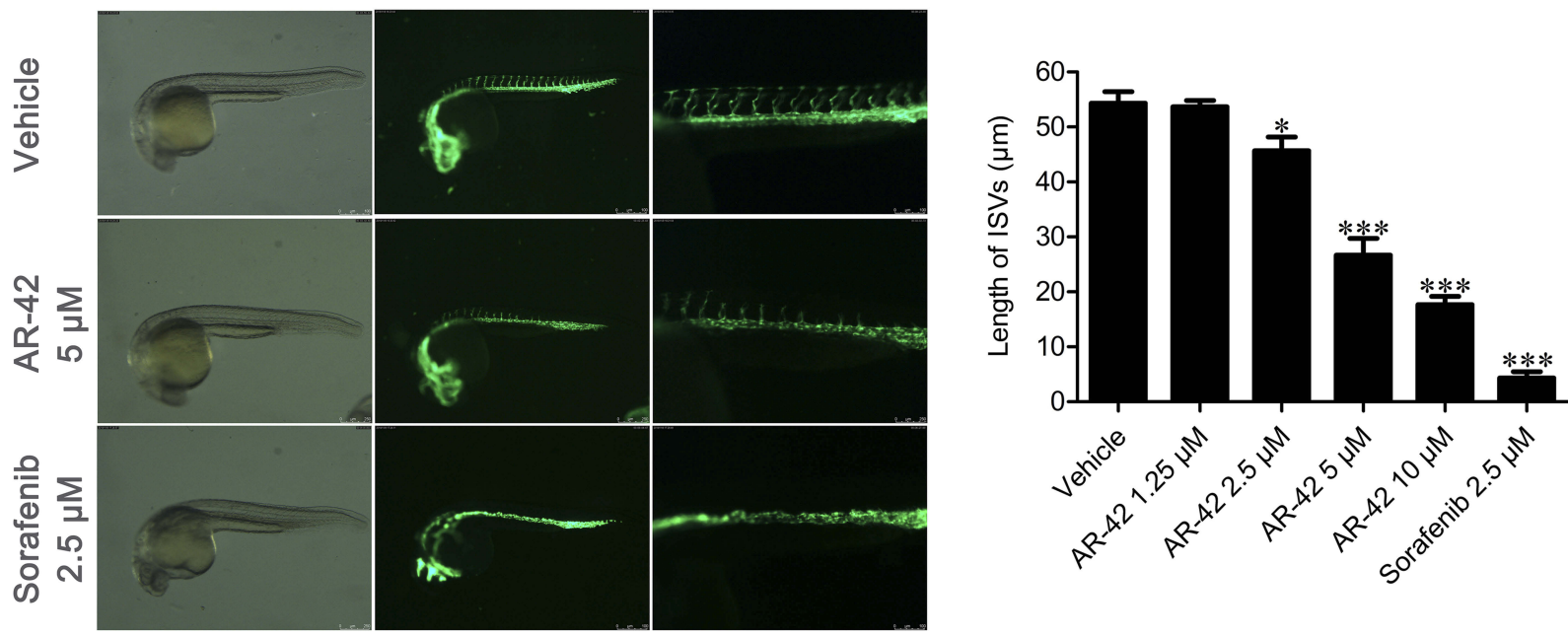

Figure 4 Anti-angiogenesis effects of AR-42. (A) Scratch assay was performed to detect HUVEC migration after treatment with the indicated concentrations of AR-42. The migrating cells are quantified for statistics. (B) Transwell chamber test was performed to detect HUVEC invasion after treatment with the indicated concentrations of AR42. The invading cells are quantified for statistics. (C) Tube formation assay was performed to detect the ability of HUVECs to aggregate into tubes after treatment with AR42. Vessel branch points are quantified for statistics. (D) Anti-angiogenesis ability of AR-42 and the positive-control sorafenib in transgenic zebrafish model. The length of intersegmental vessels (ISVs) is quantified for statistics. Values are presented as mean $\pm S D(n=3) ; * P<0.05$ vs vehicle; $* * P<0.01$ vs vehicle; $* * * P<0.00$ I vs vehicle. Abbreviations: HUVEC, human umbilical vein endothelial cell; ISV, intersegmental vessel.

at concentrations $>3 \mu \mathrm{M}$. AR-42 showed little influence on the total amount of Stat3, Akt, and ERK. Overall, these data suggest that AR-42 inhibits the growth of ESCC cells by upregulating the expression of $\mathrm{p} 21$ and blocking the transduction of multiple signaling cascades related to pro-proliferation and anti-apoptosis, especially the Stat3-mediated signaling.

\section{In vivo Antitumor Effect of AR-42 in TE-I Xenografts}

We constructed xenograft models using more sensitive TE1 cells to further assess the in vivo anti-ESCC efficacy of AR-42. As shown in Figure 6A, oral administration of AR-42 every other day for 21 days effectively inhibited tumor growth in the TE-1 xenograft model; the TGI rates of AR-42 at $25 \mathrm{mg} / \mathrm{kg}$ and $50 \mathrm{mg} / \mathrm{kg}$ were $56.3 \%$ and
$81.4 \%$, respectively. No significant difference in the weight of mice was observed among each group throughout the course of administration (Figure 6B).

After the animal experiment ended, tumor xenografts were harvested and processed for immunohistochemistry. As displayed in Figure 6C, treatment with AR-42 (50 mg/ $\mathrm{kg})$ led to a remarkable increase in the acetylation level of histone $\mathrm{H} 3$ (Lys 9) and a substantial reduction in Ki67-positive cells (proliferating tumor cells) compared with treatment with vehicle control. Meanwhile, TUNEL-positive cells (apoptotic cells) increased significantly in the AR-42 treated group (Figure 6D). In addition, CD31 immunohistochemical staining was adopted to label tumor vessels, and AR-42 could efficaciously diminish the vascular density in tumors at a dose of $50 \mathrm{mg} / \mathrm{kg}$ (Figure 6C). Taken together, AR-42 could also 


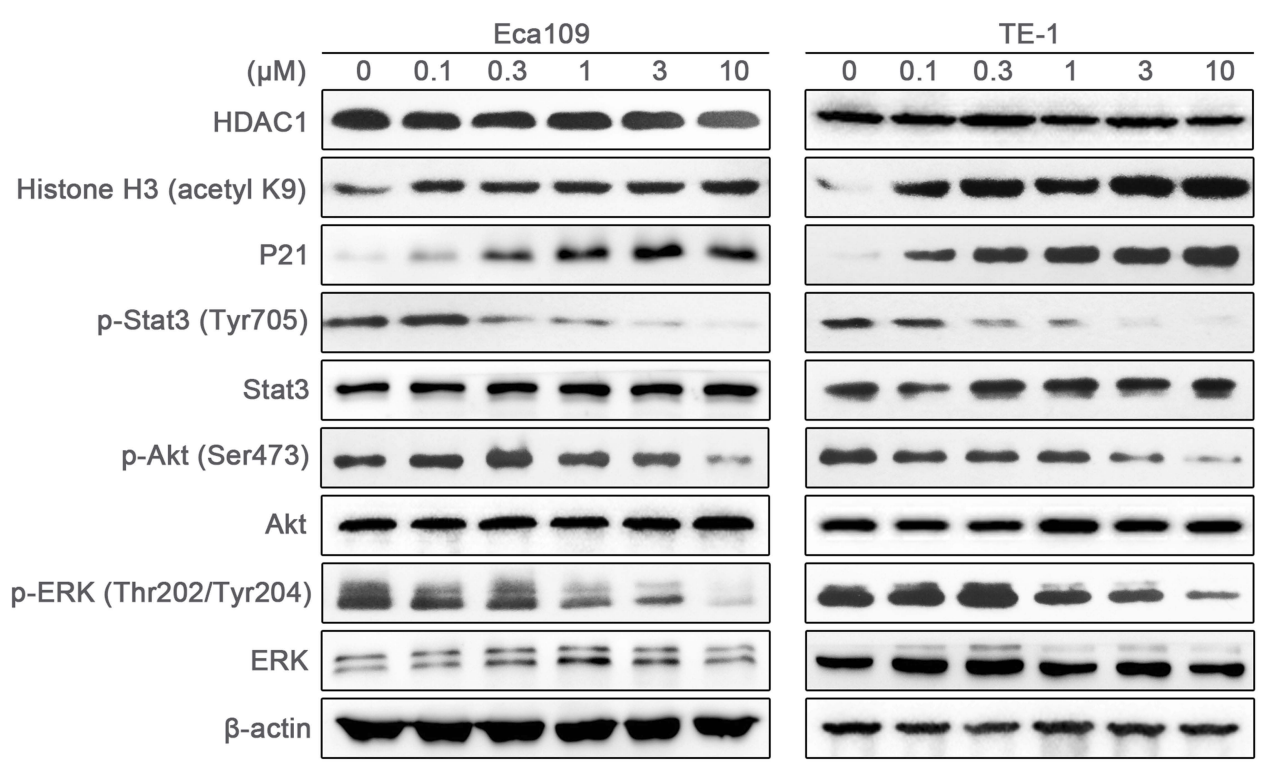

Figure 5 Western blot analysis in Ecal09 and TE-I cells treated with AR-42.

significantly inhibit HDAC activity in vivo and exhibited antiESCC activity via anti-proliferation and pro-apoptosis effects, as well as blockade of angiogenesis.

\section{Discussion}

HDACs are considered as potent targets for cancer treatment because multiple HDAC inhibitors have been tested in clinical trials, and so far, some of them have been approved for the clinical treatment of hematological malignancies. ${ }^{19,20}$ AR-42 developed by Chen et al is an orally bioavailable hydroxamate-tethered phenylbutyrate derivative with strong inhibitory activity against class I (HDAC 1, 2, 3 and 8) and class IIb (HDAC 6 and 10) HDACs. ${ }^{14-17,21}$ Although the efficacy of AR-42 against some tumors has been previously reported, its potency in ESCC treatment still lacks detailed data. The present study demonstrated that AR-42 potently restrains ESCC cell growth through proliferation inhibition and apoptosis induction. Meanwhile, AR-42 showed the ability to inhibit the migration, invasion, and tubulogenesis of HUVECs as well as intersegmental vessel formation in zebrafish, exhibiting moderate antiangiogenic activity. The inhibitory activity of AR-42 on ESCC cells and angiogenesis could also be observed in the TE-1 xenograft model. Moreover, this study showed that HDAC1-positive expression was much higher in ESCC cells than in paracancerous tissues and that elevated HDAC1 expression was closely relevant to lymph node metastasis and a more advanced TNM stage of ESCC, further supporting HDAC as a target in ESCC treatment and the pan-HDAC inhibitor AR-42 as a promising anti-ESCC agent.
HDAC inhibitors exert anti-cancer effects through various regulatory mechanisms, such as inhibition of signal transduction pathways related to tumor progression and regulation of apoptosis, autophagy, and cell cycle-related genes. ${ }^{22,23}$ The antitumor molecular mechanisms of HDAC inhibitors are not uniform; they may differ in different tumor types and depend on specific agents. ${ }^{23,24}$ The results of Western blot analysis showed that AR-42 exerts its anti-ESCC effects mainly through elevating the expression of cell cycle inhibitor $\mathrm{p} 21$ and inhibiting the activity of Stat3-mediated signaling. Blockade of Akt/mTOR and MAPK/ERK signaling cascades could just be observed in ESCC cells treated with AR-42 at high concentrations; therefore, they are not the main contributors for the potency of AR-42 against ESCC. Additionally, although it is difficult to accurately quantify the contribution of tumor cell suppression and anti-angiogenesis to anti-ESCC activity of AR-42 in vivo, the former should play a major role; this inference is based on the antiproliferative and antiangiogenic activity of AR-42 in vitro, as well as its mechanism of the anti-ESCC activity.

\section{Conclusion}

In conclusion, the present study demonstrated that HDAC1 is closely related to ESCC progression and might be a promising target for ESCC treatment. The pan-HDAC inhibitor AR-42 has significant potency for inhibiting ESCC cell growth and moderate effect in suppressing angiogenesis, displaying strong anti-ESCC effects both in vitro and in vivo. Taken together, AR-42 as a potential candidate for ESCC therapy deserves further evaluation. 
A

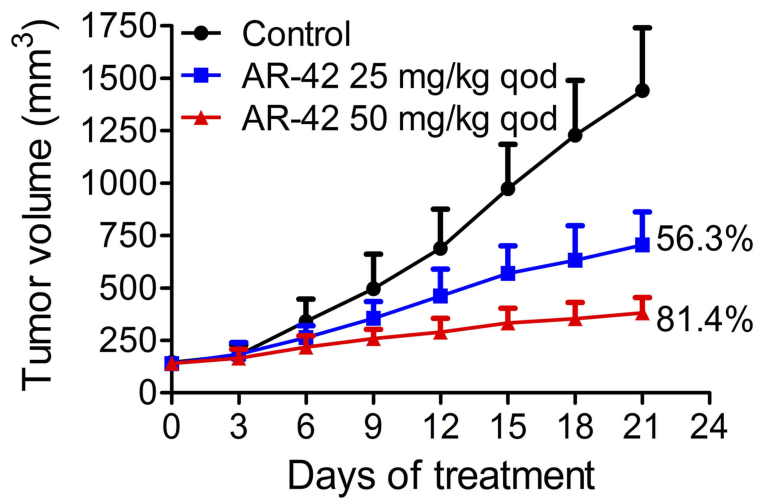

C

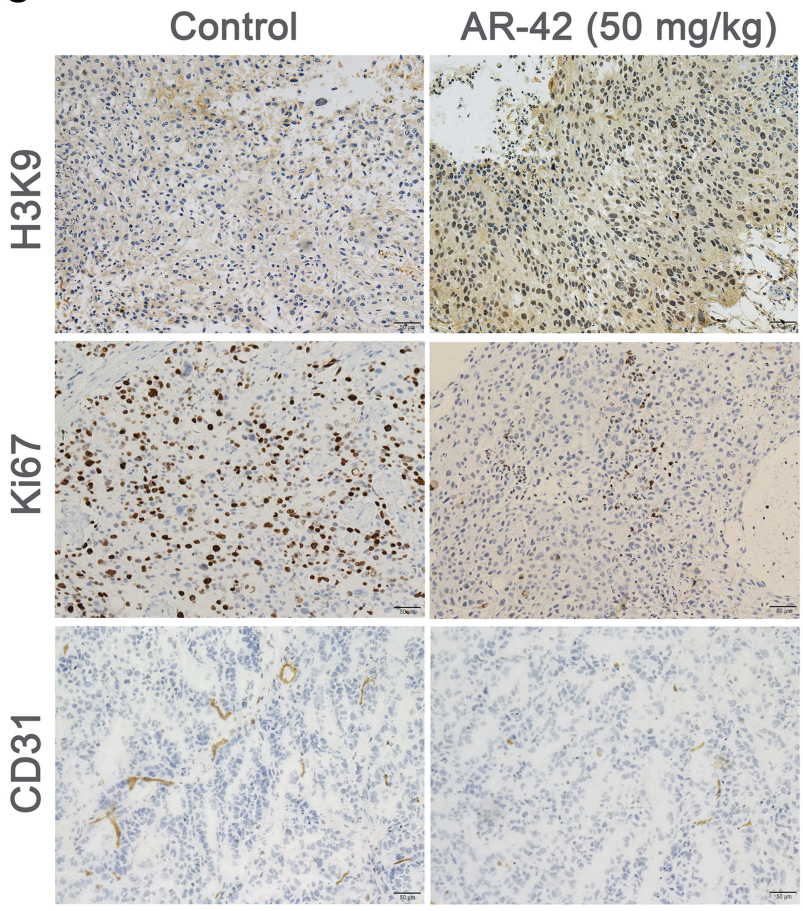

B

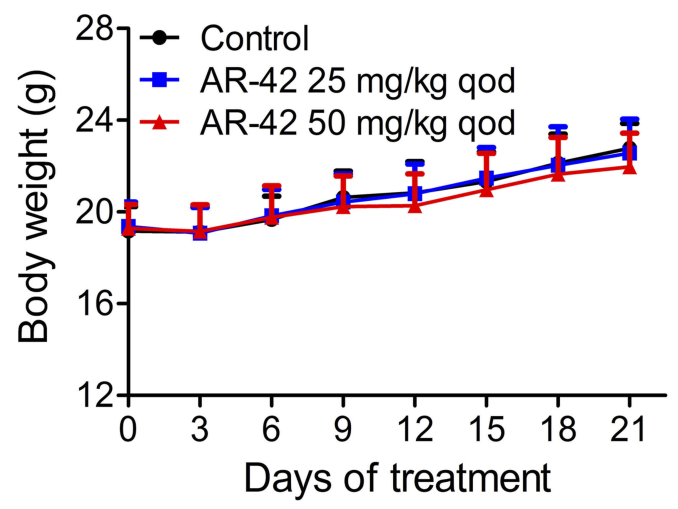

D

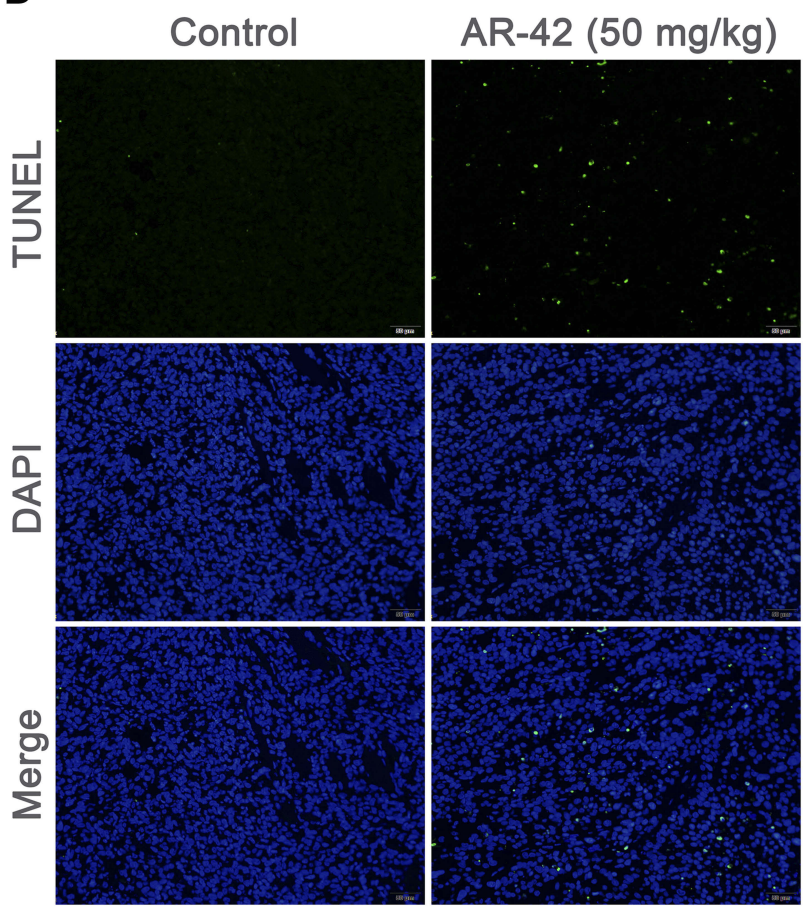

Figure 6 In vivo anti-ESCC effects of AR-42. (A) Tumor inhibition curve of AR-42 in the TE-I xenografts. Tumor volumes are presented as mean \pm SD ( $\mathrm{n}=5$ ). (B) Weight monitoring in different treatment groups of TE-I xenografts. Body weights are presented as mean $\pm S D(n=5)$. (C) TE-I xenografts treated with vehicle and 50 mg/kg AR-42 were harvested for histone $\mathrm{H} 3$ (acetyl K9) immunostaining, Ki67 proliferating cell detection and CD3I staining. Scale bars represent $50 \mu \mathrm{m}$. (D) TE-I xenografts treated with vehicle and $50 \mathrm{mg} / \mathrm{kg}$ AR-42 were harvested for TUNEL immunostaining. Scale bars represent $50 \mu \mathrm{m}$.

Abbreviations: ESCC, esophageal squamous cell carcinoma; TUNEL, TdT-mediated dUTP Nick-End Labeling.

\section{Acknowledgements}

This work was supported by the National Natural Science Foundation of China (81702286), the Applied Basic Research Fund and Fundamental Research Fund of Science \& Technology Department of Sichuan Province (2018JY0652 and 2017YSKY0001), and the Special Foundation for Young Scientists of Sichuan Academy of Medical Sciences \& Sichuan Provincial People's Hospital (2016QN11).

\section{Disclosure}

The authors report no conflicts of interest in this work.

\section{References}

1. Bray F, Ferlay J, Soerjomataram I, Siegel RL, Torre LA, Jemal A. Global cancer statistics 2018: GLOBOCAN estimates of incidence and mortality worldwide for 36 cancers in 185 countries. CA Cancer J Clin. 2018;68 (6):394-424. doi:10.3322/caac.v68.6 
2. Mimura K, Yamada L, Ujiie D, et al. Immunotherapy for esophageal squamous cell carcinoma: a review. Fukushima J Med Sci. 2018;64 (2):46-53. doi:10.5387/fms.2018-09

3. Crosby T, Hurt CN, Falk S, et al. Chemoradiotherapy with or without cetuximab in patients with oesophageal cancer (SCOPE1): a multicentre, Phase 2/3 randomised trial. Lancet Oncol. 2013;14(7):627-637. doi:10.1016/S1470-2045(13)70136-0

4. Vicente-Dueñas C, Hauer J, Cobaleda C, Borkhardt A, SánchezGarcía I. Epigenetic priming in cancer initiation. Trends Cancer. 2018;4(6):408-417. doi:10.1016/j.trecan.2018.04.007

5. Conte M, De Palma R, Altucci L. HDAC inhibitors as epigenetic regulators for cancer immunotherapy. Int J Biochem Cell Biol. 2018;98:65-74. doi:10.1016/j.biocel.2018.03.004

6. Tsilimigras DI, Ntanasis-Stathopoulos I, Moris D, Spartalis E, Pawlik TM. Histone deacetylase inhibitors in hepatocellular carcinoma: A therapeutic perspective. Surg Oncol. 2018;27(4):611-618. doi:10.1016/j.suronc.2018.07.015

7. Mishra VK, Wegwitz F, Kosinsky RL, et al. Histone deacetylase class-I inhibition promotes epithelial gene expression in pancreatic cancer cells in a BRD4- and MYC-dependent manner. Nucleic Acids Res. 2017;45(11):6334-6349. doi:10.1093/nar/gkx212

8. Bao L, Diao H, Dong N, et al. Histone deacetylase inhibitor induces cell apoptosis and cycle arrest in lung cancer cells via mitochondrial injury and p53 up-acetylation. Cell Biol Toxicol. 2016;32 (6):469-482. doi:10.1007/s10565-016-9347-8

9. Wahaib K, Beggs AE, Campbell H, Kodali L, Ford PD. Panobinostat: A histone deacetylase inhibitor for the treatment of relapsed or refractory multiple myeloma. Am J Health Syst Pharm. 2016;73 (7):441-450. doi:10.2146/ajhp150487

10. Zeng LS, Yang XZ, Wen YF, et al. Overexpressed HDAC4 is associated with poor survival and promotes tumor progression in esophageal carcinoma. Aging. 2016;8(6):1236-1249. doi:10.18632/ aging. 100980

11. Li S, Wang F, Qu Y, et al. HDAC2 regulates cell proliferation, cell cycle progression and cell apoptosis in esophageal squamous cell carcinoma EC9706 cells. Oncol Lett. 2017;13(1):403-409. doi:10.3892/ol.2016.5436

12. Song M, He G, Wang Y, Pang X, Zhang B. Lentivirus-mediated knockdown of HDAC1 uncovers its role in esophageal cancer metastasis and chemosensitivity. $J$ Cancer. 2016;7(12):1694-1700. doi: $10.7150 /$ jca. 15086
13. Chen YJ, Wang WH, Wu WY, et al. Novel histone deacetylase inhibitor AR-42 exhibits antitumor activity in pancreatic cancer cells by affecting multiple biochemical pathways. PLoS One. 2017;12(8):e0183368. doi:10.1371/journal.pone.0183368

14. Lu Q, Wang DS, Chen CS, Hu YD, Chen CS. Structure-based optimization of phenylbutyrate-derived histone deacetylase inhibitors. J Med Chem. 2005;48(17):5530-5535. doi:10.1021/ jm0503749

15. Li DR, Zhang H, Peek E, et al. Synergy of histone-deacetylase inhibitor AR-42 with cisplatin in bladder cancer. J Urol. 2015;194 (2):547-555. doi:10.1016/j.juro.2015.02.2918

16. Guzman ML, Yang N, Sharma KK, et al. Selective activity of the histone deacetylase inhibitor AR-42 against leukemia stem cells: a novel potential strategy in acute myelogenous leukemia. Mol Cancer Ther. 2014;13(8):1979-1990. doi:10.1158/1535-7163.MCT13-0963

17. Yang YT, Balch C, Kulp SK, Mand MR, Nephew KP, Chen CS. A rationally designed histone deacetylase inhibitor with distinct antitumor activity against ovarian cancer. Neoplasia. 2009;11 (6):552-563. doi:10.1593/neo.09204

18. Zhong L, Fu XY, Zou C, et al. A preclinical evaluation of a novel multikinase inhibitor, SKLB-329, as a therapeutic agent against hepatocellular carcinoma. Int J Cancer. 2014;135(12):2972-2983. doi:10.1002/ijc. 28944

19. New M, Olzscha H, La Thangue NB. HDAC inhibitor-based therapies: can we interpret the code? Mol Oncol. 2012;6(6):637-656. doi:10.1016/j.molonc.2012.09.003

20. Yoon S, Eom GH. HDAC and HDAC inhibitor: from cancer to cardiovascular diseases. Chonnam Med J. 2016;52(1):1-11. doi:10.4068/cmj.2016.52.1.1

21. Lu Q, Yang YT, Chen CS, et al. $\mathrm{Zn}^{2+}$-chelating motif-tethered short-chain fatty acids as a novel class of histone deacetylase inhibitors. $J$ Med Chem. 2004;47(2):467-474. doi:10.1021/ jm0303655

22. Dawson MA, Kouzarides T. Cancer epigenetics: from mechanism to therapy. Cell. 2012;150(1):12-27. doi:10.1016/j.cell.2012.06.013

23. Kretsovali A, Hadjimichael C, Charmpilas N. Histone deacetylase inhibitors in cell pluripotency, differentiation, and reprogramming. Stem Cells Int. 2012;2012:1-10. doi:10.1155/2012/184154

24. Eckschlager T, Plch J, Stiborova M, Hrabeta J. Histone deacetylase inhibitors as anticancer drugs. Int J Mol Sci. 2017;18(7):1414-1438. doi:10.3390/ijms 18071414

\section{Publish your work in this journal}

Drug Design, Development and Therapy is an international, peerreviewed open-access journal that spans the spectrum of drug design and development through to clinical applications. Clinical outcomes, patient safety, and programs for the development and effective, safe, and sustained use of medicines are a feature of the journal, which has also been accepted for indexing on PubMed Central. The manuscript management system is completely online and includes a very quick and fair peer-review system, which is all easy to use. Visit http://www. dovepress.com/testimonials.php to read real quotes from published authors. 